\title{
Study on the Role of Color Psychology in Children's Wear
}

\author{
Wan-jin XIE ${ }^{1}$ and Yu-mei CUI ${ }^{2, *}$ \\ ${ }^{1}$ Fashion. Art Design Institute, Donghua University, 1882, Yan An Road (w), Shanghai, \\ $\mathrm{CHN}$ \\ ${ }^{2}$ Fashion. Art Design Institute, Donghua University, 1882, Yan An Road (w), Shanghai, \\ $\mathrm{CHN}$ \\ ${ }^{*}$ Corresponding author
}

Keywords: Color Psychology, Children's Wear Design, Color Design, Clothing Psychology.

\begin{abstract}
Color psychology has been developed into an independent science and applied in practice and many designs. Color design is a key factor in children's wear design. To grasp the color design rules in children's wear, it's necessary to understand children's feelings about color, color tendency and color resonance, and grasping the color design in children's wear from the perspective of color psychology is an important topic which needs to be studied in children's wear design.
\end{abstract}

\section{Introduction}

According to the visual characteristics of mankind, color is the first thing which they have access to when they see an item and so it's with fashion design. Hence, in three elements of fashion design, color is the first attribute which can be perceived rather than shape and fabric, thus determining the important role of color in fashion design. In children's wear design, owing to the characteristics of objects, colors are more varied than those in adults' wear. For example, in order to show children's lively and native features, color matching in children's wear is bolder, brighter and jumping than adults' wear. Contrasting colors, complementary colors and similar colors, etc. are often adopted to produce strong visual stimulation. Existing studies have proved that bright and vivid colors are significant to healthy development of children's psychology. Besides, children of different age groups differ greatly in physiology and psychology, and their choices and feelings about colors show great changes as well. Hence, grasping psychological feelings brought about by colors with application principles of color psychology is an extremely crucial step in children's wear design.

\section{Visual Color Psychology Impacts Visual Feelings of Children's Wear}

According to color psychology, color is objective itself; yet, people's color psychology via what they see is subjective. It's considerably complicated changes from consciousness, thinking, will, life experience to reaction and behavior. Different colors bring people different psychological feelings. Colors can influence not only people's emotions but also their behaviors and choices.

\section{Impact of Color Tempe rature on Children's Wear}

Physical temperature in color does not exist, but different color would produce different memories and associations, which make people feel warm or cold from the colors. For instance, people can easily associates flame and sun when they see red or orange, which makes them feel the warmth. When see blue or cyan-blue, they may feel colder because 
of ocean, snow, shadows, etc. Influenced by the subjective psychology of color, people would make different choices in clothing because of different hue and different temperature, and so did the children's wear. According to studies on color psychology, children of over three years old can perceive color temperature, and show their preference in color. Compared with adults, children tend to choose warm colors. From the perspective of psychology, warm colors represent vitality, liveliness, happiness and optimism. Children who like warm colors are more lively and active and excel in communicating with people. Hence, in this aspect, colors of children's wear are more inclined to warm or neutral while steady cold colors take up a larger proportion in adults' wear. Warm colors such as red, orange and yellow are not only often used in autumn and winter clothes but also spring and summer clothes. Children's youth and vitality plus the set-off of warm colors will make fashion warmer, sweeter, livelier and safer. In children's wear design, large area of extremely cold tones should be avoided; but it doesn't mean that cold colors should not be used. Blue series such as sky blue, acid blue and sea blue are often used in boys' wear. Such blues can make children think of beautiful things such as sky, lake and beach, thus stimulating them to think and building personalities of calmness and sagacity.

\section{Impact of Color Purity on Children's Wear}

Children show strong emotional feelings about bright colors, which is determined by their physiological features. Child ren cannot feel changes in color gray scale like adults. Accordingly, bright and vivid colors can attract them and train their ability of concentration. Colors with higher purity can make children feel happy and relaxed, influence healthy development of their psychology. Therefore, compared with adults' wear, children's wear is more splendid in colors. Bright color blocks are a color matching method which is often used in children's wear and even accessories. Color purity in children's wear will change with children's age. Generally speaking, gentle colors should be selected for infants so as not to affect normal development of their eyeballs; kids who are at the innovative stage of intellectual development should wear brighter colors with more printing patterns and colors; elder kids can wear colors which are basically close to colors used in adults' wear. The purity is relatively low and neural gray colors which represent maturity and prudence are adopted.

\section{Impact of Color B rightness on Children's Wear}

In the choice of clothing color, people would like to choose the light-colored clothing in summer; it will be light, breathable and cool. While in winter, they prefer to choose dark-colored clothing that can be felt calmer and heavier. Likewise, children's wear follow this rule basically, but it is worth noting that Children's wear should be dirt-resistant. Dirt-resistant can be achieved by fabric processing and color selection, however, it is more effectively with color selection. General speaking, the color of high brightness dirties more easily than the color of low brightness. So it is available to use low brightness colors in the pocket, neckline, cuffs, bottom of trousers, etc. Through the reasonable color collocation, it can achieve the effect both beauty and function. It should also be noted that children's eyes would be in a state of tension and fatigue if they see the high brightness colors for a long time. Therefore, children's wear should avoid using large area of high brightness color such as fluorescent yellow and fluorescent orange. But in some particular functional clothing such as raincoats, it can be used to ensure the safety. 


\section{Different Physiological Ages Impact Color Psychology and Choices}

Owing to different physiological and psychological statuses, children of different physiological age groups vary in color feelings and choices. Hence, it's extremely necessary to study influences of colors over children based on different age groups.

\section{Color Psychology and Choices of Infants and Small Kids}

Eyeballs of infants are not comple tely developed. Overly bright colors will cause visual stimulation to them and affect their healthy development. While selecting colors, contrasting and gaudy colors such as fluorescence colors should be avoided. Generally speaking, choosing neutral colors, moderate brightness and purity is helpful to healthy physiological and psychological growth of infants, such as light blue, light green and light pink. Small kids of preschool age are curious about the world, studious and active. It's the key stage for their intelligence, psychology and behavioral habit. Kids at this stage are extremely sensitive about bright and vivid colors and possess strong perception of colors. Grasping children's psychological perception of colors at this stage can train their healthy and uplifting attitude. For instance, orange can attract children's attention and train their lively and optimistic character; blue can make children think calmly and with composure; green brings refreshing and clear visual feelings to children. Designers should correctly apply color psychology in fashion and choose corresponding colors according to children's occasions and emotional needs.

\section{Color psychology and Choices of Middle Kids}

Middle kids refer to children aging from six to eight years old. Children at this stage receive elementary education. Children's occasions in the school should be paid attention to in fashion color design. For example, in class, overly bright and contrasting colors usually distract children and make them unable to concentrate on what the teacher says; for the sake of safety, dull and gray colors shall not be used. Bright, lively but solemn colors can be used, such as cobalt blue and kermesinus often used in winter school uniform, light blue, light green and yellow often used in summer school uniform. At the same time, special attention should be paid to gender difference. No much difference in colors exists between infants and small kids. However, for school age children, physiological and psychological differences between different genders gradually show up. Desirable treatment of such differences can help with healthy psychological development of children.

\section{Color psychology and Choices of Elder Kids.}

Children over 9 years old have formed their own color perception and their own color preferences. Even the ability to distinguish colors has basically reached the level of adult. At this stage, children's wear is more similar with adult's wear, also the color of children's wear can reference from the color of adult's wear to a certain extent. Sometimes children's wear is a smaller version of adult's wear in the style, however, we should know that copy color collocation from adult's wear is not allowed. In elder children's wear using the lively colors, generous printing and the details of decoration can bring the vitality, healthy and energy of teenagers. The color of adult's is solemn and rigorous, which is not fit enough for kids. Using neutral and generous color such as beige, dark blue and coffee brown can produce an excellent color effect.

\section{Other Physiological Conditions Influence Color Psychology and Choices}

In addition to physiological age, other physiological features of children can impact their color choices as well. 


\section{Color Psychology and Choices of Children of Diffe rent Gende rs}

Different genders vary in many aspects such as mode of thinking, intellectual s tructure and emotional activities as well as color tendency. Except infants' wear which doesn't show obvious gender inclination, gender inclination in color design of children's wear at other stages should be paid attention to. Colors of girls' wear tend to be pink-purple with high transparency while colors of boys' wear should be blue-green and black-white with low transparency. Meanwhile, some historical factors can impact gender inclination in color design. For example, for a long time, pink has been regarded as girl's color while light blue is deemed as boy's color. As children grow, color differences become more apparent in terms of gender. Consciously differentiating genders in garment colors plays an important role in training children's gender consciousness.

\section{Color Psychology and Choices of Children of Diffe rent Skin Colors}

Children's skin color impacts color choices to a great extent as well. Correctly selecting colors which are suitable for children's skin colors can avoid shortcomings and highlight their strengths. Take Asian people for example. The skin of Asian people is a bit beige. There are many types in beige skin such as cold beige, warm beige, dull beige and fair color. In general, fashion can be used to adjust the skin color to some extent. For instance, cold beige skin can warm the skin through yellow and orange colors; warm and red skin color can be neutralized by blue colors; dull and black skin should be accompanied by bright colors with high purity instead of colors with low transparency and purity, thus setting off liveliness of fashion; children with fair skin can wear bright clothes, thus looking more delicate and pretty, and can easily control yellow series which cannot be worn by children of beige skin color.

\section{Color Psychology and Choices of Children of Diffe rent Statures}

Color psychology maintains that different colors vary in such features as close and far, light and heavy, soft and hard, strong and weak. Based on such features, defects in stature can be compensated to some extent. For instance, colors with low transparency can produce effect of shrinking and retrograding. Therefore, fat children should wear dark color clothes so as to improve visual effect while thin and weak children should wear bright-colored clothes. Generally speaking, warm colors with high transparency and purity bring with them the effect of expanding forward and outward. On the contrary, cold colors with low transparency and purity have the characteristic of shrinking backwards and inwards. Desirable use of such features only can bring about garments which are in line with children's physiological features.

\section{Summary}

All in all, a children's wear designer must learn about relations between fashion colors and children's psychology and physiology as well as influences of different colors over children from the perspective of color psychology. Color changes in children's wear are more varied than those in adults' wear. There's a great diversity of factors influencing selection of colors in children's wear, which requires designers to have the ability of considering colors and color matching of children's wear in a comprehensive way.

\section{References}

[1] Yumei Cui, Children's Wear Design, Donghua University Press, Shang Hai, 2010. 
[2] Xiaoping Liu, Analysis of The Psychological Suggestion of The Clothing Color, Popular Literature and Art. 03 (2010) 36-37.

[3] Guoqing Sun, The Clothing Choice of Children's Photography Studio, Beauty \& Times. 04 (2010) 101-103.

[4] Wenjing Zhang, The Application of Personality Psychology in The Color of Art Design, University of Qigihar (2013)

[5] Hongbo Wang, The Application of Emotion Psychology in The Color of Art Design, University of Qigihar (2013)

[6] Information on https://en.wikipedia.org/wiki/Color_psychology 